\title{
SPHERICAL FUNCTIONS ON ORTHOGONAL GROUPS
}

\author{
YASUHIRO KAJIMA
}

\section{Introduction}

Let $G$ be a $p$-adic connected reductive algebraic group and $K$ a maximal compact subgroup of $G$. In [4], Casselman obtained the explicit formula of zonal spherical functions on $G$ with respect to $K$ on the assumption that $K$ is special. It is known (Bruhat and Tits [3]) that the affine root system of algebraic group which has good but not special maximal compact subgroup is $A_{1}, C_{2}$, or $B_{n}(n>3)$, and all $B_{n}$-types can be realized by orthogonal groups. Here the assumption "good" is necessary for the Satake's theory of spherical functions.

Thus in this paper we write down explicitly the zonal spherical functions on all $\mathfrak{p}$-adic (we assume that $\mathfrak{p}$ does not lie over 2 for the convenience of calculation) orthogonal groups but the case of even dimensional split orthogonal groups (this case is contained in the work of Casselman) and determine the image of Satake transform. To do so, we use Macdonald's idea by which he has obtained explicit formula for the $\mathfrak{p}$-adic Chevalley group.

Now we recall briefly some basic notion of Satake transform. Let $\mathrm{L}(G)$ be the set of all compactly supported continuous functions on $G$ with values in $\mathbf{C}$. We put

$$
L(G, K)=\left\{f \in L(G) \mid f\left(u g u^{\prime}\right)=f(g) \text { for all } u, u^{\prime} \in K, g \in G\right\} .
$$

For $f_{1}, f_{2} \in L(G, K)$, we define their product by the convolution

$$
\left(f_{1} * f_{2}\right)(g)=\int_{G} f_{1}\left(g g_{1}^{-1}\right) f_{2}\left(g_{1}\right) d g_{1}
$$

where $g \in G$, and $d g_{1}$ is the bi-invariant Haar measure on $G$ normalized by the condition that the volume of $K$ is equal to 1 . The multiplication gives the structure

Received March 12, 1993.

Revised February 24, 1995. 
of $\mathbf{C}$-algebra to $L(G, K)$. The algebra $L(G, K)$ is called local Hecke algebra of $G$ with respect to $K$. A complex-valued function $\omega$ is called a zonal spherical function if $\omega$ is continuous, not identically zero, satisfies the condition that

$$
\omega\left(g_{1}\right) \omega\left(g_{2}\right)=\int_{K} \omega\left(g_{1} k g_{2}\right) d k,
$$

for all $g_{1}, g_{2} \in G$. Now the Satake transform of $f \in L(G, K)$ with respect to a zonal spherical function $\omega$ is given by

$$
f \rightarrow \hat{f}=\int_{G} \omega f d g .
$$

It is known that the transform above is an isomorphism from $L(G, K)$ onto a polynomial ring. Since each element of $L(G, K)$ is written as a finite sum of the characteristic functions of sets $K g K$ for $g \in G$ and the zonal spherical function is constant on $K g K$, we have only to know the explicit formulae of the zonal spherical functions and the volume of $K g K$ to obtain explicit formula of Satake transform. We determine the explicit formulae of the zonal spherical functions in Section 2 except for even dimensional split orthogonal groups and calculate the volume $K g K$ in Section 3.

Notation. Let $k$ be a $\mathfrak{p}$-adic field where $\mathfrak{p}$ does not lie over 2 . We denote the maximal order in $k$ by $\mathfrak{D}$ and its prime ideal by $\mathfrak{p}=(\Pi)$. We denote by $I_{n}$ the identity matrix of degree $n$. For a topological group $G$, we denote the volume of a set $S \subset G$ for a given Haar measure by $\operatorname{vol}_{G}(S)$. We omit the index $G$ in $\operatorname{vol}_{G}(S)$ if there is no confusion.

\section{§1. Definitions and properties of fundamentals}

1.1. Let $V$ be a right vector space over $k$ of dimension $n$, and $\langle$, a non-degenerate symmetric bilinear form on $V$ with Witt index $\nu$. We put $n_{0}=n$ $-2 \nu$. In this paper we treat only the case where $n_{0} \neq 0$ as mentioned in the introduction. There exists (not uniquely determined) a system of vectors $\left\{e_{i}, e_{i}^{\prime}\right\}$ $(1 \leq i \leq \nu)$ such that

$$
\left\langle e_{i}, e_{j}\right\rangle=\left\langle e_{i}^{\prime}, e_{j}^{\prime}\right\rangle=0\left\langle e_{i}, e_{j}^{\prime}\right\rangle=\delta_{i j} \text { for all } i, j,
$$

( $\delta_{i j}$ is Kronecker's symbol). Put

$$
V_{0}=\left(\sum e_{i} k+\sum e_{i}^{\prime} k\right)^{\perp}, L_{0}=\left\{x \in V_{0} \mid\langle x, x\rangle \in \mathfrak{o}\right\}, L=\sum e_{i} \mathrm{o}+\sum e_{i}^{\prime} \mathrm{o}+L_{0} .
$$


Then $V_{0}$ is anisotropic and $L$ is a maximal lattice of $V$. There is an orthogonal system of vectors $\left\{f_{i}\right\}\left(1 \leq i \leq n_{0}\right)$ such that

(1) $\quad L_{0}=\sum_{i=0}^{n_{0}} f_{i} \mathrm{D}, \operatorname{ord}_{\mathfrak{p}}\left(\left\langle f_{1}, f_{1}\right\rangle\right) \leq \operatorname{ord}_{\mathfrak{p}}\left(\left\langle f_{2}, f_{2}\right\rangle\right) \leq \cdots \leq \operatorname{ord}_{\mathfrak{p}}\left(\left\langle f_{n_{0}}, f_{n_{0}}\right\rangle\right)$.

We define $\alpha$ as the non-negative integer such that

$$
\operatorname{ord}_{\mathfrak{p}}\left(\left\langle f_{\alpha}, f_{\alpha}\right\rangle\right)=\left\langle\operatorname{ord}_{\mathfrak{p}}\left(\left\langle f_{\alpha+1}, f_{\alpha+1}\right\rangle\right)=1\right.
$$

We also put

$$
\beta=n_{0}-\alpha
$$

Hereafter we fix this basis $\left\{e_{1}, \ldots, e_{\nu}, f_{1}, \ldots, f_{n_{0}}, e_{\nu}^{\prime}, \ldots, e_{1}^{\prime}\right\}$ of $V$ and identify the algebra $\operatorname{End}_{k}(V)$ with the matrix algebra $M_{n}(k)$. Then the symmetric bilinear form $\langle$,$\rangle is represented by the matrix$

$$
\left.S=\left(\begin{array}{c|c|c}
0 & 0 & \tilde{I}_{\nu} \\
\hline 0 & S_{0} & 0 \\
\hline \tilde{I}_{\nu} & 0 & 0
\end{array}\right)\right\} n_{0},
$$

where we set

$$
S_{0}=\operatorname{diag}\left(\left\langle f_{1}, f_{1}\right\rangle, \ldots,\left\langle f_{n_{0}}, f_{n_{0}}\right\rangle\right) \in M_{n_{0}}(k), \tilde{I}_{\nu}=\left({ }_{1} \cdot{ }^{1}\right) .
$$

By the well-known theory of quadratic forms on local fields, we may assume that $S_{0}$ is one of the following matrices:

$$
\begin{aligned}
& \left(n_{0}=1\right) \quad(1),(\pi),(u),(u \pi)
\end{aligned}
$$

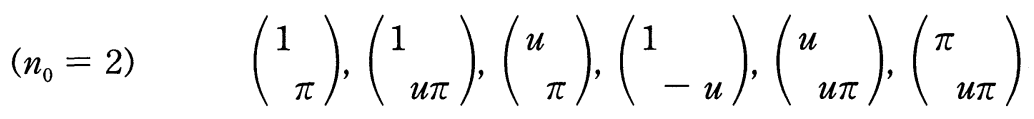

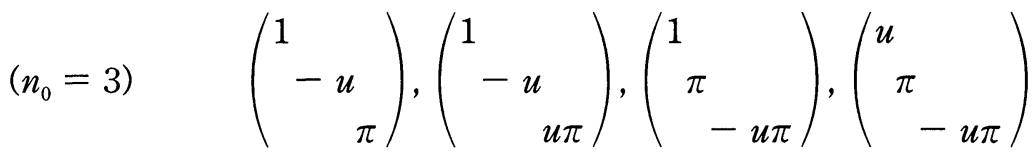

$$
\begin{aligned}
& \left(n_{0}=4\right) \quad\left(\begin{array}{ccc}
1 & & \\
-u & \\
& \pi & \\
& & -u \pi
\end{array}\right) \text {, }
\end{aligned}
$$

where $u$ is a representative of $o^{x} /\left(o^{x}\right)^{2}$. Let $G$ and $G_{0}$ be orthogonal groups of 
similitudes of $V$ and $V_{0}$ respectively, with respect to $\langle$,$\rangle , that is,$

$$
\begin{aligned}
& G=\left\{\left.g \in G L(V)\right|^{t} g S g=\mu(g) S\right\} \\
& G_{0}=\left\{\left.g_{0} \in G L\left(V_{0}\right)\right|^{t} g_{0} S_{0} g_{0}=\mu_{0}\left(g_{0}\right) S_{0}\right\} .
\end{aligned}
$$

As in [7] we define three subgroups $K, H$, and $N$ as follows:

$$
K:=\{k \in G \mid k L=L\}=\left\{k \in G \mid k, k^{-1} \in G L(n, \mathfrak{o})\right\}
$$

(6) $H:=\left(\operatorname{diag}\left(\xi_{1}, \ldots, \xi_{\nu}, h_{0}, \mu_{0}\left(h_{0}\right) \xi_{\nu}^{-1}, \ldots, \mu_{0}\left(h_{0}\right) \xi_{1}^{-1}\right) \in G \mid \xi_{\imath} \in k^{\times}, h_{0} \in G_{0}\right\}$

$$
N:=\left\{n=\left(\begin{array}{c|c|c}
A & 0 & 0 \\
\hline * & I_{n_{0}} & 0 \\
\hline * & * & \tilde{I}_{\nu}^{t} A^{-1} \tilde{I}_{\nu}
\end{array}\right) \in G\right\}
$$

where $A$ is a lower unipotent triangular matrix. This $K$ is a good maximal compact subgroup of $G$. In fact, $K$ is a special good maximal compact subgroup of $G$ except for the case $n_{0}=\beta \leq 2$. However, it is known that $K$ is not special in the case of $n_{0}=\beta \leq 2$. In other words

Remark 1. The maximal compact subgroup defined above is good but not special if and only if $(\alpha, \beta)=(0,1)$ or $(0,2)$.

We define the symbol $e_{0}$ and the group $M$ by

$$
\operatorname{ord}_{\mathfrak{p}} \mu(G)=\frac{2}{e_{0}} \mathbf{Z} . \quad M=\mathbf{Z}^{\nu} \times \frac{2}{e_{0}} \mathbf{Z} .
$$

It is easy to see that $e_{0}=2$ if $\alpha=\beta$, and $e_{0}=1$ if $\alpha \neq \beta$. For $(m)=\left(m_{1}, \ldots\right.$, $\left.m_{\nu}, \frac{m_{0}}{e_{0}}\right) \in M$, we define $\Pi^{(m)}$ as follows:

$$
\Pi^{(m)}=\operatorname{diag}\left(\Pi^{m_{1}}, \ldots, \Pi^{m_{\nu}}, w^{m_{0}}, \mu_{0}(w)^{m_{0}} \Pi^{-m_{\nu}}, \ldots, \mu_{0}(w)^{m_{0}} \Pi^{-m_{1}}\right),
$$

where $w$ denotes an arbitrarily fixed element of $G_{0}$ such that $\operatorname{ord}_{p} \mu_{0}(w)=\frac{2}{e_{0}}$. We denote by $D$ the subgroup in $H$ generated by $\Pi^{(m)}$, for $(m) \in M$. We consider any character $s$ of $D$ as a character of $H$ by putting $s(h)=1$ for all $h \in H_{0}=H \cap$ $G L(n, \mathrm{o})$. It is known ([7]),

$$
G=K H K=K D K(\text { Cartan decomposition) }
$$




$$
=K H N=K D N(\text { Iwasawa decomposition). }
$$

We define the modular function $\delta$ on $H$ by

$$
d\left(h n h^{-1}\right) / d(n)=\delta^{-1}(h) .
$$

For a character $s$ of $D$, we define a function $\phi_{s}$ on $G$ by

$$
\phi_{s}(k h n)=s(h) \delta^{1 / 2}(h) \quad(k \in K, h \in H, n \in N) .
$$

Then the zonal spherical function $\omega_{s}$ on $G$ is defined to be

$$
\omega_{s}\left(x^{-1}\right):=\int_{K} \phi_{s}(x k) d k, \quad(x \in G)
$$

where the Haar measure $d k$ on $K$ is normalized such that $\operatorname{vol}(K)=1$. It is trivial that $\phi_{s}$ is a multiplicative function on $H$. Since any $h^{\prime} \in H$ normalizes $N$, we have

$$
\phi_{s}\left(x h^{\prime}\right)=\phi_{s}\left(k h n h^{\prime}\right)=\phi_{s}\left(k h h^{\prime} n^{\prime}\right)=\phi_{s}(h) \phi_{s}\left(h^{\prime}\right)=\phi_{s}(x) \phi_{s}\left(h^{\prime}\right),
$$

where $x=k h n, n^{\prime}=h^{\prime-1} n h^{\prime}$.

1.2. Now we prepare some notion. The group $K, H$, and $N$, are the same as defined in Section 1.1. In a $\nu$-dimensional vector space $\mathbf{R}^{\nu}$ with standard basis $\left\{\varepsilon_{1}, \ldots, \varepsilon_{\nu}\right\}$, the root system $\sum$ of $B_{\nu}$-type is given by

$$
\Sigma=\left\{ \pm \varepsilon_{i}, \pm \varepsilon_{i} \pm \varepsilon_{j} \mid 1 \leq i, j \leq \nu, i \neq j\right\} .
$$

A set of positive roots and a set of simple roots of $\Sigma$ are given as follows:

$$
\begin{gathered}
\Sigma^{+}=\left\{\varepsilon_{i}, \varepsilon_{j}+\varepsilon_{k}, \varepsilon_{j}-\varepsilon_{k} \mid 1 \leq i \leq \nu, 1 \leq j<k \leq \nu\right\}, \\
\left\{\varepsilon_{\nu}, \varepsilon_{i}-\varepsilon_{i+1}(1 \leq i \leq \nu-1)\right\} .
\end{gathered}
$$

Also define $\Sigma^{-}=\Sigma \backslash \Sigma^{+}$. For any simple root $\alpha \in \Sigma^{+}$, we define an isometry $w_{\alpha}$ of $\mathbf{R}^{\nu}$, which we call simple reflection, by

$$
w_{a}(a)=-a, \text { and } w_{a}\left(\Sigma^{+} \backslash a\right)=\Sigma^{+} \backslash a .
$$

We define the Weyl group as the group $W$ generated by $w_{a}, a \in \Sigma^{+}$. Now let $w=w_{1} \ldots w_{r} \in W$ be a "reduced" word, that is to say, $w_{i}$ 's are simple reflections, and $w$ is not a product of $r^{\prime}$ simple reflections for $r^{\prime}<r$. We call $r$ the "length" of $w$ and denote it by $l(w)$. It is known that if $w=w_{1} \ldots w_{r-1} w_{r}$ is a reduced word where $w_{r}=w_{a}$ for a simple root $\alpha \in \sum$, than we have

$$
\left(w_{1} \ldots w_{r-1}\right) a \in \Sigma^{+} \text {and } w a=\left(w_{1} \ldots w_{r-1}\right)(-a) \in \Sigma^{-}
$$


(cf. [8, p.216]). For a simple root $a$, we define automorphism $w^{(a)}$ on $M$ and $w(a)$ $\in G L(V)$ as follows:

$$
\begin{gathered}
w^{\left(\varepsilon_{i}-\varepsilon_{i+1}\right)}:=\left[\begin{array}{l}
m_{i} \rightarrow m_{i+1}, m_{i+1} \rightarrow m_{i} \\
m_{j} \rightarrow m_{j}(j \neq i, i+1),
\end{array}\right. \\
w^{\left(\varepsilon_{\nu}\right)}:=\left[\begin{array}{l}
m_{\nu} \rightarrow-m_{\nu}+\frac{2}{e_{0}} m_{0}, \\
m_{j} \rightarrow m_{j}(j \neq \nu) .
\end{array}\right. \\
:=\left(e_{1}, \ldots, e_{i+1}, e_{\nu}, \ldots, e_{\nu}, f_{1}, \ldots, f_{n_{0}}, e_{\nu}^{\prime}, \ldots, e_{\imath}^{\prime}, e_{i+1}^{\prime}, \ldots, e_{1}\right) . \\
\left(e_{1}, \ldots, e_{\nu}, f_{1}, \ldots, f_{n_{0}}, e_{\nu}^{\prime}, \ldots, e_{1}^{\prime}\right) w\left(\varepsilon_{\nu}\right) \\
:=\left(e_{1}, \ldots, e_{\nu-1}, e_{\nu}^{\prime},-f_{1}, f_{2}, \ldots, f_{n_{0}}, e_{\nu}, e_{\nu-1}^{\prime}, \ldots, e_{1}^{\prime}\right) .
\end{gathered}
$$

Denote by $W_{M}$ (resp. $W_{V}$ ) the group generated by $w^{(a)}$ (resp. $w(a)$ ). Then $W \simeq W_{M}$ $\simeq W_{V}$, and we identify them if there is no confusion. It is easy to see that

$$
w \Pi^{(m)} w^{-1}=\Pi^{(w(m))} \text { for } w \in W .
$$

Thus, since $W \subset K$, we can take

$$
x=\Pi^{(m)} \in D,(m)=\left(m_{1}, \ldots, m_{\nu}, \frac{m_{0}}{e_{0}}\right) \text { with } m_{1} \geq \ldots \geq \frac{m_{0}}{e_{0}}
$$

as the representatives of double cosets $K \backslash G / K$. Therefore we have only to evaluate $\omega_{s}(x)$ for $x \in D$ of the form above.

Now we define nilpotent matrices $M\left( \pm \varepsilon_{i} \pm \varepsilon_{j}\right)(t)$, and $M_{h}\left( \pm \varepsilon_{i}\right)(t)(1 \leq h$ $\leq n_{0}$ ) for $t \in k$ as follows:

$$
\begin{aligned}
& M\left(\varepsilon_{i}-\varepsilon_{j}\right)(t)=\left(m_{r s}\right), m_{r s}=\left[\begin{array}{rl}
t & \text { if } r=i, s=j, \\
-t & \text { if } r=n+1-j, s=n+1-i, \\
0 & \text { otherwise. }
\end{array}\right. \\
& M\left(\varepsilon_{i}+\varepsilon_{j}\right)(t)=\left(m_{r s}\right), m_{r s}=\left[\begin{array}{rl}
t & \text { if } r=i, s=n+1-j, \\
-t & \text { if } r=j, s=n+1-i, \\
0 & \text { otherwise. }
\end{array}\right. \\
& M\left(-\varepsilon_{i}-\varepsilon_{j}\right)(t)={ }^{t} M\left(\varepsilon_{i}+\varepsilon_{j}\right)(t) . \\
& M_{h}\left(\varepsilon_{i}\right)(t)=\left(m_{r s}\right), \quad m_{r s}=\left[\begin{array}{cl}
-t\left\langle f_{h}, f_{h}\right\rangle & \text { if } r=i, s=\nu+h, \\
t & \text { if } r=\nu+h, s=n+1-i, \\
0 & \text { otherwise. }
\end{array}\right.
\end{aligned}
$$




$$
M_{h}\left(-\varepsilon_{i}\right)(t)=\left(m_{r s}\right), m_{r s}=\left[\begin{array}{cl}
-t\left\langle f_{h}, f_{h}\right\rangle & \text { if } r=n+1-i, s=\nu+h \\
t & \text { if } r=\nu+h, s=i \\
0 & \text { otherwise. }
\end{array}\right.
$$

Also define $\exp M$ for nilpotent matrices $M \in M_{n}(k)$ by

$$
\exp M=1+\frac{M}{1}+\frac{M^{2}}{2 !}+\cdots
$$

Now we define subgroups $U_{h}^{\prime}\left( \pm \varepsilon_{i}\right), U_{\left( \pm \varepsilon_{i}\right)}, U_{\left( \pm \varepsilon_{i} \pm \varepsilon_{j}\right)}$ of $G$ by

$$
\begin{gathered}
U_{h}^{\prime}\left( \pm \varepsilon_{i}\right):=\left\{\exp M_{h}\left( \pm \varepsilon_{i}\right)(t) \mid t \in k\right\} \\
U_{\left(\varepsilon_{i}\right)}:=U_{1}^{\prime}\left(\varepsilon_{i}\right) \cdots U_{n_{0}}^{\prime}\left(\varepsilon_{i}\right), U_{\left(-\varepsilon_{i}\right)}:=U_{1}^{\prime}\left(-\varepsilon_{i}\right) \cdots U_{n_{0}}^{\prime}\left(-\varepsilon_{\imath}\right) \\
U_{\left( \pm \varepsilon_{i} \pm \varepsilon_{j}\right)}:=\left\{\exp M\left( \pm \varepsilon_{i} \pm \varepsilon_{j}\right)(t) \mid t \in k\right\} .
\end{gathered}
$$

It is easy to see that for some fixed $h \in\{1, \ldots, \nu\}, U_{h}^{\prime}\left(\varepsilon_{\imath}\right)$ commute with each other, which implies that $U_{\left(\varepsilon_{i}\right)}$ (resp. $\left.U_{\left(-\varepsilon_{i}\right)}\right)$ is a group. The fact that $U_{\left( \pm \varepsilon_{i} \pm \varepsilon_{j}\right)}$ are groups is trivial.

Remark 2. The $(i+\nu, n+1-\nu)$-entry $\left(1 \leq i \leq n_{0}\right)$ of $u=u_{1} \cdots u_{n_{0}} \in$ $U_{\left(\varepsilon_{\nu}\right)}$ is the same as that of $u_{\imath}$.

For $u=\left(u_{k l}\right) \in U_{\left(\varepsilon_{i}\right)}$ (resp. $\left.U_{\left(-\varepsilon_{i}\right)}\right)$, we put

$$
\operatorname{ord}_{\mathfrak{p}} u=\operatorname{ord}_{\mathfrak{p}}\left(u_{i, n+1-i}\right)\left(\operatorname{resp} . \operatorname{ord}_{\mathfrak{p}} u=\operatorname{ord}_{\mathfrak{p}}\left(u_{n+1-i, i}\right)\right) \text {. }
$$

Similarly we define $\operatorname{ord}_{\mathfrak{p}} u$ for $u=\exp M\left( \pm \varepsilon_{\imath} \pm \varepsilon_{j}\right)(t)$ by

$$
\operatorname{ord}_{\mathfrak{p}} u=\operatorname{ord}_{\mathfrak{p}} t \text {. }
$$

We define

$$
U_{(a)}(l):=\left\{u \in U_{(a)} \mid \operatorname{ord}_{\mathfrak{p}} u \geq l\right\} .
$$

Then $U_{(a)}(l),\left(a \in \sum\right)$ are subgroups and we have for all $a \in \sum, w \in W$

$$
w U_{(a)}(l) w^{-1}=U_{(w a)}(l), \text { and } w U_{(a)} w^{-1}=U_{(w a)} .
$$

For $l \geq 0$, we define $U^{+}(l)$ (resp. $U^{-}(l)$ ) to be the subgroup of $K$ generated by $U_{(a)}(l), a \in \Sigma^{+}$(resp. $U_{(a)}(l), a \in \Sigma^{-}$), i.e.,

$$
U^{+}(l):=\left\langle U_{(a)}(l) \mid a \in \Sigma^{+}\right\rangle, U^{-}(l):=\left\langle U_{(a)}(l) \mid a \in \Sigma^{-}\right\rangle .
$$

Moreover we define

$$
U^{+}:=\left\langle U_{(a)} \mid a \in \Sigma^{+}\right\rangle
$$


It is easy to see that $U^{+}(0) \cdot H_{0} \cdot U^{-}(1)=H^{0} \cdot U^{+}(0) \cdot U^{-}(1)$ is a subgroup of $K$ where $H_{0}=H \cap K$, which we denote by $B$ :

$$
B:=U^{+}(0) \cdot H_{0} \cdot U^{-}(1)=H_{0} \cdot U^{+}(0) \cdot U^{-}(1) .
$$

Now we write down some groups defined above explicitly.

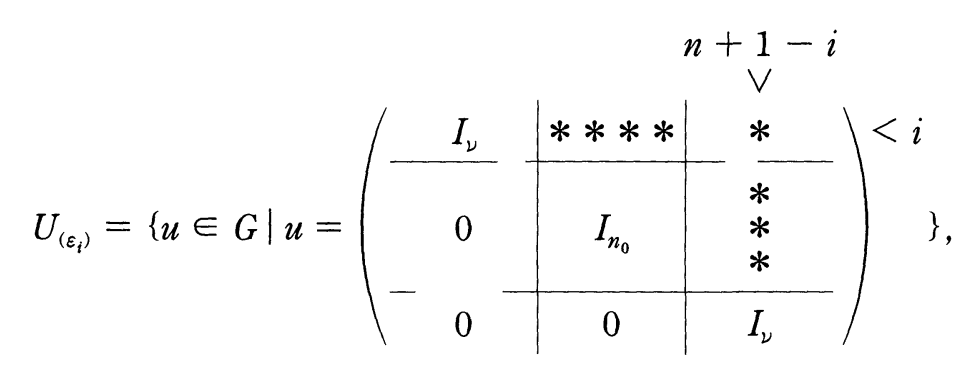

where diagonal blocks are identity matrices, $*$ 's are on the $i$-th row or the $(n+$ $1-i)$-th column, and entries in the $1 \times 3$ block is zero except for $(i, n+1-$ i)-entry, other entries are zero.

$$
\begin{aligned}
& U^{+}(l)=\left\{u=\left(\begin{array}{c|c|c}
A^{+} & C^{+} & D^{+} \\
\hline 0 & I_{n_{0}} & E^{+} \\
\hline 0 & 0 & B^{+}
\end{array}\right) \in G \mid A^{+}-I_{\nu}, B^{+}-I_{\nu}, D^{+}, \in M_{\nu}\left(p^{l}\right)\right\}, \\
& U^{-}(l)=\left\{u=\left(\begin{array}{c|c|c}
A^{-} & 0 & 0 \\
\hline C^{-} & I_{n_{0}} & 0 \\
\hline D^{-} & E^{-} & B^{-}
\end{array}\right) \in G \mid A^{-}-I_{\nu}, B^{-}-I_{\nu}, D^{-}, \in M_{\nu}\left(p^{l}\right)\right\},
\end{aligned}
$$

where $A^{+}$and $B^{+}$(resp. $A^{-}$and $B^{-}$) are upper (resp. lower) unipotent triangular matrices.

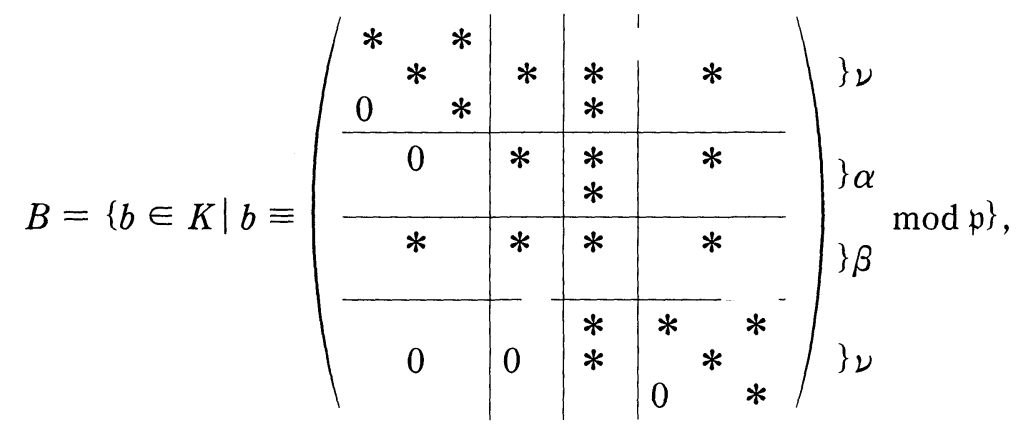

where $(1,1)$ and $(4,4)$ blocks are upper triangular matrices, $(2,1),(4,1)$ and $(4,2)$ blocks are zero matrices. (It turns out that $(1,3),(2,3)$, and $(4,3)$ blocks are congruent to zero matrices $\bmod \mathfrak{p}$.) 
Remark 3. Put, $U_{i}^{\prime}\left(\varepsilon_{\nu}\right)(l):=U_{i}^{\prime}\left(\varepsilon_{\nu}\right) \cap U_{\left(\varepsilon_{\nu}\right)}(l)$, and

$$
u=u_{1} \ldots u_{n_{0}} \in U_{\left(\varepsilon_{\nu}\right)}(l) \text { (decomposition in (17)). }
$$

Since $\left\langle e_{\nu}^{\prime}, e_{\nu}^{\prime}\right\rangle=0$, we have

$$
\left\langle f_{1} x_{1}+\cdots+f_{n_{0}} x_{n_{0}}, f_{1} x_{1}+\cdots+f_{n_{0}} x_{n_{0}}\right\rangle=-2 y
$$

where $y$ is the $(\nu, n+1-\nu)$-entry of $u$, and $x_{i}$ is $(\nu+i, n+1-\nu)$-entry of $u_{i}$. This equality implies [3, Theorem (10.1.15), Proposition (10.1.12)] that

$$
\operatorname{ord}_{\mathfrak{p}}\left\langle f_{i} x_{\imath}, f_{i} x_{i}\right\rangle \geq \operatorname{ord}_{\mathfrak{p}} y \geq l
$$

for all $i\left(1 \leq i \leq n_{0}\right)$. Since $-\left\langle f_{i} x_{i}, f_{i} x_{i}\right\rangle / 2$ is the $(\nu, n+1-\nu)$-entry of $u_{i}$, we have

$$
u_{i} \in U_{i}^{\prime}\left(\varepsilon_{\nu}\right)(l) \text { for all } i \text {. }
$$

Therefore

$$
\left[U_{\left(\varepsilon_{\nu}\right)}(l): U_{\left(\varepsilon_{\nu}\right)}(m)\right]=\prod_{i=1}^{n_{0}}\left[U_{i}^{\prime}\left(\varepsilon_{\nu}\right)(l): U_{i}^{\prime}\left(\varepsilon_{\nu}\right)(m)\right]
$$

Now we state the following theorem of Hijikata which is used later.

ThEOREM H (Hijikata [5, p.33]). The maximal compact subgroup $K$ is decomposed as follows:

$$
K=\bigcup_{w \in W} B w B \text { (disjoint union). }
$$

Remark 4. In [5] Hijikata proved the Bruhat decomposition in more general situation. But we use Hijikata's theorem in restricted form as above. In our case, the Theorem $\mathrm{H}$ is proved by straightforward calculation.

Now as the Lemma 2.6.2 in [6], we know

$$
\begin{gathered}
U^{+}(l)=\prod_{a \in \Sigma^{+}} U_{(a)}(l), \text { and } U^{+}=\prod_{a \in \Sigma^{+}} U_{(a)}, \\
\text { (resp. } \left.U^{-}(l)=\prod_{a \in \Sigma^{-}} U_{(a)}(l), \text { and } U^{-}=N=\prod_{a \in \Sigma^{-}} U_{(a)}\right)
\end{gathered}
$$

where we can take the products above in an arbitrary order. Moreover, for any simple root $a \in \Sigma^{+}$and $l \geq 0$, we put

$$
U_{+}^{(a)}(l):=\prod_{b \in \Sigma^{+} \backslash\{a\}} U_{(b)}(l), U_{+}^{(a)}:=\prod_{b \in \Sigma^{+} \backslash\{a\}} U_{(b)},
$$




$$
U_{-}^{(-a)}(l):=\prod_{b \in \Sigma^{-} \backslash\{-a\}} U_{(b)}(l) \text {, and } U_{-}^{(-a)}:=\prod_{b \in \Sigma^{-} \backslash\{-a\}} U_{(b)}
$$

Then these groups are written as semidirect product as follows:

$$
\begin{gathered}
U^{+}(l)=U_{(a)}(l) \cdot U_{+}^{(a)}(l), U^{+}=U_{(a)} \cdot U_{+}^{(a)}, \\
U^{-}(l)=U_{(a)}(l) \cdot U_{-}^{(-a)}(l), \text { and } U^{-}=U_{(a)} \cdot U_{-}^{(-a)},
\end{gathered}
$$

where $U_{+}^{(a)}(l), U_{+}^{(a)}, U_{-}^{(-a)}(l)$, and $U_{-}^{(-a)}$ are normal subgroups of $U^{+}(l), U^{+}$, $U^{-}(l)$, and $U^{-}$, respectively. Thus we have

$$
\begin{gathered}
d u^{+}=d u_{(a)} \cdot d u_{+}^{(a)}=d u_{+}^{(a)} d u_{(a)}, \\
d u_{-}=d u_{(-a)} \cdot d u_{-}^{(-a)}=d u_{-}^{(-a)} d u_{(-a)}
\end{gathered}
$$

for any simple root $a \in \Sigma^{+}$. In the following, we assume that

$$
\operatorname{vol}\left(U^{+}(0)\right)=\operatorname{vol}\left(U_{(a)}(0)\right)=\operatorname{vol}\left(U_{+}^{(a)}(0)\right)=1 .
$$

Now define the modular function $\delta_{a}(h)$ for $h \in H$, and $a \in \sum$ by

$$
\delta_{a}(h)=d\left(h u_{(a)} h^{-1}\right) / d u_{(a)} .
$$

Then it is easy to see that

$$
\delta_{a}(h) \cdot \delta_{(-a)}(h)=1 \text {, and } \delta_{a}(h) \cdot \delta_{a}\left(w_{a} h w_{a}^{-1}\right)=1,
$$

for any simple root $a \in \Sigma^{+}$. Also define

$$
\delta^{a}(h)=d\left(h u_{+}^{(a)} h^{-1}\right) / d u_{+}^{(a)},
$$

for $h \in H$, and $a \in \Sigma^{+}$. Then we have

$$
\delta^{a}(h) \cdot \delta_{a}(h)=\delta(h)
$$

for any simple root $a \in \Sigma^{+}$.

Now for any simple root $a \in \Sigma^{+}, x \in \Pi^{(m)}$, and $w_{a} \in W$, we have

$$
\begin{aligned}
& d\left(\left(w_{a} x w_{a}^{-1}\right) u^{+}\left(w_{a} x w_{a}^{-1}\right)^{-1}\right) \\
= & d\left(\left(w_{a} x w_{a}^{-1}\right) u_{(a)}\left(w_{a} x w_{a}^{-1}\right)^{-1}\right) \cdot d\left(\left(w_{a} x w_{a}^{-1}\right) u_{+}^{(a)}\left(w_{a} x w_{a}^{-1}\right)^{-1}\right) \\
= & \delta_{a}(x)^{-1} d u_{(a)} \cdot d\left(\left(w_{a} x w_{a}^{-1}\right) u_{+}^{(a)}\left(w_{a} x w_{a}^{-1}\right)^{-1}\right) \\
= & \delta_{a}(x)^{-1} d u_{(a)} \cdot d\left(x u_{+}^{(a)} x^{-1}\right)=\delta_{a}(x)^{-1} \cdot d u_{(a)} \delta^{a}(x) d u_{+}^{(a)} \\
= & \delta_{a}^{-2}(x) \cdot \delta(x) \cdot d u^{+} .
\end{aligned}
$$

Since (33) is equal to $\delta\left(w_{a} x w_{a}^{-1}\right) d u^{+}$by definition, we have 


$$
\delta\left(w_{a} x w_{a}^{-1}\right) / \delta(x)=\left(\delta_{a}(x)\right)^{-2} .
$$

\section{\$2. Calculations of spherical functions}

2.1. We now calculate the zonal spherical function $\omega_{s}\left(x^{-1}\right)$. We put $V_{w}=$ $\operatorname{vol}(B w B)$ where $\operatorname{vol}(K)=1$. For the convenience of notation, we define

$$
\begin{gathered}
\Pi_{i}:=\Pi^{(m(t))} \in D,(m(i)) \in M \\
\Pi\left(\varepsilon_{i}-\varepsilon_{i+1}\right):=\Pi_{i} \text { and } \Pi\left(\varepsilon_{\nu}\right):=\Pi_{\nu}
\end{gathered}
$$

where $m(\nu)=\left(0_{\nu-1}, 1,0\right), m(i)=\left(0_{t-1}, 1,-1,0_{\nu-i}\right)$ if $i \neq \nu$. (Here $0_{i}$ is a zero matrix in $M(1, i)$.) Let $x=\Pi^{(m)}$, such that

$$
(m)=\left(m_{1}, \ldots, m_{\nu}, \frac{m_{0}}{e_{0}}\right) \text { with } m_{1} \geq \cdots \geq m_{\nu} \geq \frac{1}{e_{0}} m_{0} .
$$

Note that $x$ above satisfies the conditions

$$
x \cdot U^{+}(r) \cdot x^{-1} \subset U^{+}(r), x \cdot U_{(a)}(r) \cdot x^{-1} \subset U_{(a)}(r)
$$

and

$$
x \cdot U_{(-a)}(r) x^{-1} \supset U_{(-a)}(r)
$$

for any positive root $a \in \Sigma^{+}$and any integer $r$.

Morever it is easy to see that

$$
w^{-1} \cdot U^{-}(1) \cdot w \subset B \text {, and } w^{-1} \cdot H_{0} \cdot w=H_{0} \subset B
$$

for every $w \in W\left(H_{0}=H \cap K\right)$. Thus we have from the decomposition (19),

$$
B \cdot w \cdot B=U^{+}(0) \cdot w \cdot B \text {. }
$$

Now for a character $s$ of $D$, the value of the zonal spherical function $\omega_{s}$ in Section 1 at $x=\Pi^{(m)}$ above is

$$
\begin{gathered}
\omega_{s}\left(x^{-1}\right)=\int_{K} \phi_{s}(x k) d k=\sum_{w \in W} \int_{B w B} \phi_{s}(x k) d k \quad \text { (by Theorem H) } \\
=\sum_{w \in W} V_{w} \cdot \int_{B} \phi_{s}(x w b) d b,
\end{gathered}
$$

(by (38) and (39), using $x U^{+}(0) x^{-1} \subset U^{+}(0) \subset K$,) where $d b$ is normalized as $\operatorname{vol}(B)=1$. Then we have 


$$
\omega_{s}\left(x^{-1}\right)=\sum_{w \in W} V_{w} \cdot \int_{U^{+}(0)} \phi_{s}\left(x w u^{+}\right) d u^{+}
$$

where $\operatorname{vol}\left(U^{+}(0)\right)=1$. Putting $x^{\prime}=w^{-1} x w$, we have

$$
\begin{gathered}
\int_{U^{+}(0)} \phi_{s}\left(x w u^{+}\right) d u^{+}=\int_{U^{+}(0)} \phi_{s}\left(w^{-1} x w u^{+}\right) d u^{+} \\
=\int_{U^{+}(0)} \phi_{s}\left(x^{\prime} u^{+} x^{\prime-1} x^{\prime}\right) d u^{+}=\left(s \delta^{1 / 2}\right)\left(x^{\prime}\right) \int_{U^{+}(0)} \phi_{s}\left(x^{\prime} u^{+} x^{\prime-1}\right) d u^{+} \\
=\left(s \delta^{1 / 2}\right)\left(x^{\prime}\right) \delta^{-1}\left(x^{\prime}\right) \int_{x^{\prime} U^{+}(0) x^{\prime-1}} \phi_{s}\left(u^{+}\right) d u^{+}=\left(s \delta^{-1 / 2}\right)\left(x^{\prime}\right) \cdot \int_{x^{\prime} U^{+}(0) x^{\prime-1}} \phi_{s}\left(u^{+}\right) d u^{+}
\end{gathered}
$$

Here for any character $\chi$ of $D$ and $w \in W$, we define a character $w(\chi)$ (or simply $w \chi$ ) of $D$ by

$$
w \chi(d):=\chi\left(w^{-1} d w\right)
$$

Then $(42)=w\left(s \delta^{-1 / 2}\right)(x) \int_{x^{\prime} U^{+}(0) x^{\prime-1}} \phi_{s}\left(u^{+}\right) d u^{+}$. We denote this by $J_{w}(s)$, i,e.,

$$
J_{w}(s):=w\left(s \delta^{-1 / 2}\right)(x) \int_{x^{\prime} U^{+}(0) x^{\prime-1}} \phi_{s}\left(u^{+}\right) d u^{+}\left(=\int_{U^{+}(0)} \phi_{s}\left(x w u^{+}\right) d u^{+}\right)
$$

Then we have from (41)

$$
\omega_{s}\left(x^{-1}\right)=\sum_{w \in W} V_{w} \cdot J_{w}(s)
$$

Remark 5. Since $U^{+}(0)$ is compact, $K$ is open, and $G=K H N$, there exists a finite set $\left\{h_{i} n_{i}\right\} \subset H N$ such that $x^{\prime} U^{+}(0) x^{\prime-1}$ is contained in the union of the sets $K h_{i} n_{i}$. Thus we have

$$
J_{w}(s)=w\left(s \delta^{-1 / 2}\right)(x) \cdot \sum_{i} \operatorname{vol}\left(K h_{i} n_{i} \cap x^{\prime} U^{+}(0) x^{\prime^{-1}}\right) \cdot s \delta^{1 / 2}\left(h_{i}\right)
$$

where $\operatorname{vol}\left(U^{+}(0)\right)=1$. In other words, $J_{w}(s) \subset \mathbf{R}\left[(s)\left(\Pi_{1}\right)^{ \pm 1}, \ldots,(s)\left(\Pi_{\nu}\right)^{ \pm 1}\right]$.

The computation of $w_{s}\left(x^{-1}\right)$ is reduced to the integrals $J_{w}(s), w \in W$. Let us continue the calculation. If $w=1$ (= identity), we have

$$
J_{w}(s)=J_{1}(s)=\left(s \delta^{1 / 2}\right)(x)
$$

using the fact that $x U^{+}(0) x^{-1} \subset K$ (by (37)). Now assume $w \neq 1$. Then there exists a simple root $a \in \Sigma^{+}$such that 


$$
w a \in \Sigma^{-}, w=w^{\prime} w_{a} \text { with } l(w)=l\left(w^{\prime}\right)+1 .
$$

Then $x^{\prime} U^{+}(0) x^{\prime-1}$ can be written as a semi-direct product of

$$
Z^{a}:=x^{\prime} U_{+}^{(a)}(0) x^{\prime^{-1}} \text { and } Z_{a}:=x^{\prime} U_{(a)}(0) x^{\prime^{-1}}
$$

uniquely. Therefore we have

$$
\begin{gathered}
J_{w}(s)=w\left(s \delta^{-1 / 2}\right)(x) \cdot \int_{Z^{a}} \int_{Z_{a}} \phi_{s}\left(u^{a} u_{a}\right) d u_{+}^{a} d u_{a} \\
=\left(w\left(s \delta^{-1 / 2}\right)(x)\right)\left(\int_{Z^{a}} \int_{U_{(a)}} \phi_{s}\left(u^{a} u_{a}\right) d u_{+}^{a} d u_{a}-\int_{Z^{a}} \int_{U_{(a)} \backslash Z_{a}} \phi_{s}\left(u^{a} u_{a}\right) d u_{+}^{a} d u_{a}\right) .
\end{gathered}
$$

Putting

$$
\begin{gathered}
J_{w}^{1}(s)=w\left(s \delta^{-1 / 2}\right)(x) \int_{Z^{a}} \int_{U_{(a)}} \phi_{s}\left(u^{a} u_{a}\right) d u_{+}^{a} d u_{a}, \\
J_{w}^{0}(s)=w\left(s \delta^{-1 / 2}\right)(x) \int_{Z^{a}} \int_{U_{(a)} \backslash Z_{a}} \phi_{s}\left(u^{a} u_{a}\right) d u_{+}^{a} d u_{a},
\end{gathered}
$$

we have

$$
J_{w}(s)=J_{w}^{1}(s)-J_{w}^{0}(s) .
$$

Now we define $C_{0}(a, s)$ for any simple root $a \in \Sigma^{+}$by

$$
C_{0}(a, s):=\int_{U_{(a)}} \phi_{s}\left(u_{a}\right) d u_{a}
$$

We did not care the convergence of integrals, but it is easy to see that if $|s(\Pi(a))|>1$ for a simple root $a$ (see (36)), then $C_{0}(a, s)$ and all integrals are absolutely convergent, and so, our calculations above are justified.

2.2. In this section, we give relations of $J_{w}, J_{w}^{0}, J_{w}^{1}$, and $C_{0}(a, s)$. We prepare several lemmas.

Lemma 1. In the notation as before, we have

$$
J_{w^{\prime}}(s)=\left(w^{\prime} s\right)(x)\left(w \delta^{-1 / 2}\right)(x) \int_{w_{a} Z^{a} w_{a}^{-1}} \phi_{s}\left(u^{a}\right) d u_{+}^{a},
$$

where $Z^{a}=x^{\prime} U_{+}^{a}(0) x^{\prime-1}, x^{\prime}=w^{-1} x w, w=w^{\prime} w_{a}$.

Proof. We put $x_{1}=w^{\prime-1} x w^{\prime}=w_{a} x^{\prime} w_{a}^{-1}$. Since $w_{a}^{-1} U_{+}^{a}(0) w_{a}=U_{+}^{a}(0)$ (see (18) and (24)), we have 


$$
w_{a} Z^{a} w_{a}^{-1}=w_{a} \cdot x^{\prime} U_{+}^{a}(0) x^{\prime-1} \cdot w_{a}^{-1}=x_{1} U_{+}^{a}(0) x_{1}^{-1} .
$$

Since $w^{\prime} a \in \Sigma^{+}$, we have $w^{\prime} U_{a}(0) w^{\prime-1}=U_{w^{\prime} a}(0) \subset U^{+}(0)$ (by (18)). Thus

$$
w_{1} U_{a}(0) x_{1}^{-1} \subset w^{\prime-1} x U^{+}(0) x^{-1} w^{\prime} \subset w^{-1} U^{+}(0) w^{\prime} \subset K,
$$

where we used (37). Therefore we have

$$
\begin{gathered}
J_{w^{\prime}}(s)=w^{\prime}\left(s \delta^{-1 / 2}\right)(x) \int_{x_{1} U_{a}(0) x_{1}^{-1}} \int_{x_{1} U_{+}^{a}(0) x_{1}^{-1}} \phi_{s}\left(u^{a} u_{a}\right) d u_{a} d u_{+}^{a} \\
\left.=w^{\prime}\left(s \delta^{-1 / 2}\right)(x) \int_{x_{a} Z^{a} x_{a}^{-1}} \phi_{s}\left(u^{a}\right) d u_{+}^{a} \times \text { (volume of } x_{1} U_{a}(0) x_{1}^{-1}\right) .
\end{gathered}
$$

Since $\operatorname{vol}\left(U_{(a)}(0)\right)=1, \operatorname{vol}\left(U_{+}^{(a)}(0)\right)=1$ and $\operatorname{vol}\left(x_{1} U_{(a)}(0) x_{1}^{-1}\right)=\delta_{a}\left(x_{1}\right)=$ $d\left(x_{1} u_{(a)} x_{1}^{-1}\right) / d\left(u_{(a)}\right)$ (see (29)), we have

$$
\begin{aligned}
(54) & =w^{\prime}\left(s \delta^{-1 / 2}\right)(x) \cdot \delta^{1 / 2}\left(x_{1}\right) \delta^{-1 / 2}\left(w_{a}^{-1} x_{1} w_{a}\right) \int_{w_{a} z^{a} w_{a}^{-1}} \phi_{s}\left(u^{a}\right) d u^{a} \\
& =\left(w^{\prime} s\right)(x)\left(w \delta^{-1 / 2}\right)(x) \int_{w_{a} z^{a} w_{a}^{-1}} \phi_{s}\left(u^{a}\right) d u^{a}\left(\text { by } w^{\prime} w_{a}=w\right) .
\end{aligned}
$$

Lemma 2. Let $a \in \Sigma^{+}$be a simple root, i.e., $a=\varepsilon_{i}-\varepsilon_{i+1}$ or $\varepsilon_{\nu}$. Then $a$ non-zero element $u$ of $U_{(a)}$ is decomposed in $G$ as follows:

$$
u=h \cdot y \cdot w_{a}(\Pi(a))^{r} \cdot z, \quad \text { for } y, z \in U_{(-a)}(r)
$$

(see (36)), where $r=-\operatorname{ord}_{\mathfrak{p}} u, h \in H_{0}=H \cap K$.

Proof. The lemma is easily proved as the proof of $G=U H N$ in Satake [7]. We only note the following decomposition for the case where $S_{0}=\left(\begin{array}{ll}\Pi & -u \Pi\end{array}\right)$, and $n=4$.

$$
\begin{aligned}
& \left(\begin{array}{cccc}
1 & -\Pi a & \Pi u b & A \\
& 1 & 0 & a \\
& & 1 & b \\
& & & 1
\end{array}\right)=\left(\begin{array}{cccc}
A \Pi^{\prime} & & & \\
& h 1 & h 2 & \\
& h 3 & h 4 & \\
& & & \left(A \Pi^{\prime}\right)^{-1}
\end{array}\right)\left(\begin{array}{cccc}
1 & & & \\
\Pi^{\prime} a & 1 & \\
-\Pi^{\prime} b & 0 & 1 \\
A \Pi^{\prime \prime} & -\Pi^{\prime} \Pi a & -\Pi^{\prime} \Pi u b & 1
\end{array}\right) \\
& \times\left(\begin{array}{cccc} 
& & & 1 \\
& -1 & & \\
& & 1 &
\end{array}\right)\left(\begin{array}{ccccc}
\Pi^{r} & & & \\
& 1 & & \\
& & 1 & \\
& & & \Pi^{r}
\end{array}\right)\left(\begin{array}{ccccc}
1 & & & \\
a / A & 1 & & \\
b / A & 0 & 1 & \\
1 / A & -\Pi a / A & \Pi u b / A & 1
\end{array}\right),
\end{aligned}
$$


where $\quad A=\Pi\left(u b^{2}-a^{2}\right) / 2, \Pi^{\prime}=\Pi^{r}, \Pi^{\prime \prime}=\Pi^{2 r}, h 1=-\Pi a^{2} / A-1, h 2=-$ $\Pi a b u / A, h 3=-\Pi a b / A$, and $h 4=1-\Pi u b^{2} / A$.

Lemma 3. In the notation as above, we have

$$
\int_{U_{(a)} \backslash Z_{a}} \phi_{s}\left(u_{a}\right) d u_{a}=\left(C_{0}(a, s)-1\right) \cdot((w s)(x))^{-1} \cdot\left(\left(w^{\prime} s\right)(x)\right) .
$$

Proof. For $u_{a} \in U_{(a)} \backslash U_{(a)}(0)$, we have from Lemma 2,

$$
u_{a}=h \cdot y \cdot w_{a} \cdot(\Pi(a))^{r} \cdot z,
$$

where $h \in H \cap K, y, z \in U_{(-a)}$, Since $\operatorname{ord}_{\mathfrak{p}} u_{a}<0$, we have $\operatorname{ord}_{\mathfrak{p}} y=\operatorname{ord}_{\mathfrak{p}} z=$ - $\operatorname{ord}_{\mathfrak{p}} u_{a}>0$, and hence $y, z \in U_{(-a)}(0) \subset K$. It is easy to see that

$$
x^{\prime} h x^{\prime-1} \in K,
$$

and we have $b:=w(-a) \in \Sigma^{+}$by virtue of the choice of $w((46))$. Thus recalling (18) and (37), we have

$$
x^{\prime} y x^{\prime^{-1}}=w^{-1} x w y w^{-1} x^{-1} w \subset w^{-1} x U_{(b)}(0) x^{-1} w \subset w^{-1} U^{+}(0) w \subset K .
$$

From (56), (57), and $w_{a}^{-1} x^{\prime} w_{a} \in H$, we have

$$
\begin{gathered}
\phi_{s}\left(x^{\prime} u_{a} x^{\prime-1}\right)=\phi_{s}\left(w_{a}^{-1} x^{\prime} w_{a}(\Pi(a))^{r} x^{\prime-1}\right) \\
\left.=\phi_{s}\left((\Pi(a))^{r}\right) \cdot \phi_{s}\left(\left(w_{a}^{-1} x^{\prime} w_{a}\right) x^{\prime-1}\right)\right)=\phi_{s}\left(u_{a}\right) \cdot \phi_{s}\left(w_{a}^{-1} x^{\prime} w_{a}\right) \cdot \phi_{s}\left(x^{\prime^{-1}}\right) \\
=\phi_{s}\left(u_{a}\right)((w s)(x))^{-1} \cdot\left(\left(w^{\prime} s\right)(x)\right) \cdot \delta^{-1 / 2}\left(x^{\prime}\right) \delta^{1 / 2}\left(w_{a} x^{\prime} w_{a}^{-1}\right) \\
=\phi_{s}\left(u_{a}\right)((w s)(x))^{-1} \cdot\left(\left(w^{\prime} s\right)(x)\right) \cdot \delta_{a}^{-1}\left(x^{\prime}\right)(\mathrm{by}(34)) .
\end{gathered}
$$

By (59), we have

$$
\begin{aligned}
& \int_{U_{(a)} \backslash Z_{a}} \phi_{s}\left(u_{a}\right) d u_{a}=\delta_{a}\left(x^{\prime}\right) \times \int_{U_{(a) \backslash U_{a}(0)}} \phi_{s}\left(x^{\prime} u_{a} x^{\prime^{-1}}\right) d u_{a} \\
& \int_{U_{(a)} \backslash U_{a}(0)} \phi_{s}\left(u_{a}\right) d u_{a} \cdot((w s)(x))^{-1} \cdot\left(\left(w^{\prime} s\right)(x)\right) .
\end{aligned}
$$

Now since $\operatorname{vol}\left(U_{(a)}(0)\right)=\mathbf{1}$, we get our lemma from $(60)$.

Now we give explicit formulae of $\left.C_{0}(a, s)\right)$ for orthogonal groups. Except for the case $\alpha=0, C_{0}(a, s)$ is known by Casselman [4].

THEOREM 1. We keep the notation as above. We put $q=|\mathrm{o} / \mathfrak{p}|$, and $S_{i}=s\left(\Pi_{\imath}\right)$, 
where $s_{i}$ is a character of $D$, and $\Pi_{i}$ is defined in (35) as before. We have

i) $\quad C_{0}\left(\varepsilon_{\imath}-\varepsilon_{i+1}, s\right)=\frac{q-S_{i}}{q-q S_{i}}$.

ii) $\quad C_{0}\left(\varepsilon_{\nu}, s\right)=1+\frac{T}{1-S_{\nu}^{2}}$ where $T=\frac{\left(q^{\beta}-1\right)}{q^{(\alpha+\beta) / 2}} S_{\nu}+\frac{q^{\beta}\left(q^{\alpha}-1\right)}{q^{(\alpha+\beta)}} S_{\nu}^{2}$.

Proof. Consider the case where $a=\varepsilon_{\nu}$. Using Lemma 3 for the case where $w^{\prime}=1, w=w_{a}$, and $x=\Pi^{(m)},(m)=(1, \ldots, 1,0)$, we get

$$
\int_{U_{(a)} \backslash U_{a}(-2)} \phi_{s}\left(u_{a}\right) d u_{a}=\left(C_{0}(a, s)-1\right) S_{\nu}^{2}
$$

Thus we have

$$
\begin{aligned}
\int_{U_{a}(-2) \backslash U_{a}(0)} \phi_{s}\left(u_{a}\right) d u_{a} & =\int_{U_{a} \backslash U_{a}(0)} \phi_{s}\left(u_{a}\right) d u_{a}-\int_{U_{a} \backslash U_{\left.a^{(}-2\right)}} \phi_{s}\left(u_{a}\right) d u_{a} \\
& =\left(C_{0}(a, s)-1\right)\left(1-S_{\nu}^{2}\right) .
\end{aligned}
$$

Now from (21),

$$
\left[U_{\left(\varepsilon_{\nu}\right)}(i): U_{\left(\varepsilon_{\nu}\right)}(i+1)\right]=\prod_{j=1}^{n_{0}}\left[U_{j}^{\prime}\left(\varepsilon_{\nu}\right)(i): U_{j}^{\prime}\left(\varepsilon_{\nu}\right)(i+1)\right] .
$$

It is easy to see that if $1 \leq j \leq \alpha$ (resp. $\left.\alpha+1 \leq j \leq n_{0}=\alpha+\beta\right)$, then

$$
\begin{gathered}
{\left[U_{j}^{\prime}\left(\varepsilon_{\nu}\right)(i): U_{j}^{\prime}\left(\varepsilon_{\nu}\right)(i+1)\right]=\left[\begin{array}{ll}
q & \text { if } i \text { is even, } \\
1 & \text { if } i \text { is odd, }
\end{array}\right.} \\
\left(\text { resp. }\left[U_{j}^{\prime}\left(\varepsilon_{\nu}\right)(i): U_{j}^{\prime}\left(\varepsilon_{\nu}\right)(i+1)\right]=\left[\begin{array}{ll}
1 & \text { if } i \text { is even, } \\
q & \text { if } i \text { is odd, }
\end{array}\right)\right.
\end{gathered}
$$

which implies

$$
\left[U_{\left(\varepsilon_{\nu}\right)}(i): U_{\left(\varepsilon_{\nu}\right)}(i+1)\right]=\left[\begin{array}{ll}
q^{\alpha} & \text { if } i \text { is even } \\
q^{\beta} & \text { if } i \text { is odd }
\end{array}\right.
$$

Moreover from Lemma 2, we have for $u \in U_{(a)}$,

$$
u=h \cdot y \cdot w_{a} \cdot(\Pi(a))^{r} \cdot z, \text { for } y, z \in U_{(-a)}(r),
$$

where $r=-\operatorname{ord}_{\mathfrak{p}} u$, and $h \in H_{0}=H \cap K$. Thus, if $u \in U_{(a)} \backslash U_{(a)}(0)$, then we have $y \in K$, and $h \cdot y \cdot w_{a} \in K$. Since $z \in N$, we have $\phi_{s}(u)=\left(s \delta^{1 / 2}\right)\left((\Pi(a))^{r}\right)$, which implies 


$$
\int_{U_{a}(-2) \backslash U_{a}(0)} \phi_{s}\left(u_{a}\right) d u_{a}=\left(s \delta^{1 / 2}\right)(\Pi(a))\left(q^{\beta}-1\right)+\left(s^{2} \delta\right)(\Pi(a))\left(q^{\alpha+\beta}-q^{\beta}\right) .
$$

Since $\delta(\Pi(a))=q^{-(\alpha+\beta)}$, we have proved our theorem for the case where $a=\varepsilon_{\nu}$. For the case where $a=\varepsilon_{i}-\varepsilon_{i+1}$, it is proved similarly.

Remark 6. If $s=\delta^{-1 / 2}, a=\varepsilon_{\nu}$, then

$$
\int_{U_{a}(-2) \backslash U_{a}(0)} \phi_{s}\left(u_{a}\right) d u_{a}=\left(q^{\beta}-1\right)+\left(q^{\alpha+\beta}-q^{\beta}\right)=q^{\alpha+\beta}-1=\delta(\Pi(a))^{-1}-1=S_{\nu}^{2}-1 .
$$

This implies $C_{0}\left(\varepsilon_{\nu}, \delta^{-1 / 2}\right)=0$. Similarly, we have $C_{0}\left(\varepsilon_{i}-\varepsilon_{i+1}, \delta^{-1 / 2}\right)=0$.

It follows from Theorem 1 that, for $w \in W, C_{0}(a, s)=C_{0}(w a, w s)$ if both $a$ and $w a$ are simple roots. Therefore we can define $C_{0}(a, s)$ for any root $a$ by

$$
C_{0}(a, s)=C_{0}(w a, w s) .
$$

LEMMA 4. In the notation as before, we have

$$
\int_{Z^{a}} \int_{U_{(a)} \backslash Z_{a}} \phi_{s}\left(u^{a} u_{a}\right) d u_{+}^{a} d u_{a}=\int_{Z^{a}} \phi_{s}\left(u^{a} w_{a}\right) d u_{+}^{a} \times \int_{U_{(a)} \backslash Z_{a}} \phi_{s}\left(u_{a}\right) d u_{a},
$$

where $Z^{a}=x^{\prime} U_{+}^{(a)}(0){x^{\prime}}^{-1}, Z_{a}=x^{\prime} U_{(a)}(0) x^{\prime-1}$, and $w a \in \Sigma^{-}$.

Proof. Suppose $a=\varepsilon_{\nu}$. Define $l$ by $l=\operatorname{ord}_{\mathfrak{p}} x_{n-\nu+1}^{\prime}-\operatorname{ord}_{\mathfrak{p}} x_{\nu}^{\prime}$, where $x_{n-\nu+1}^{\prime}$ (resp. $\left.x_{\nu}^{\prime}\right)$ is $(n-\nu+1, n-\nu+1)$-entry (resp. $(\nu, \nu)$-entry) of $x^{\prime}$. It is clear that $l=\operatorname{ord}_{\mathrm{p}} u_{a}-\operatorname{ord}_{\mathrm{p}} x^{\prime} u_{a} x^{\prime-1}$ for $u_{a} \in U_{a} u \neq I_{n}$. Since $x^{\prime}=w^{-1} x w$ and $w(a) \in \Sigma^{-}$, we have $l \geq 0$ and $Z_{a}=U_{(a)}(-l)$. From Lemma $2, u_{a} \in U_{(a)} \backslash Z_{a}=$ $U_{(a)} \backslash U_{(a)}(-l)$ can be written as follows:

$$
u_{a}=h \cdot y \cdot w_{a} \cdot\left(\Pi_{\nu}\right)^{r} \cdot z
$$

where $h \in H_{0}=H \cap K, 0 \leq l<r=\operatorname{ord}_{\mathfrak{p}} y=\operatorname{ord}_{\mathfrak{p}} z=-\operatorname{ord}_{\mathfrak{p}} u_{a}$ and $y, z \in$ $U_{(a)}(r) \subset K$. Since $h$ normalize the group $Z^{a}$, we have

$$
\int_{Z^{a}} \int_{U_{(a)} \backslash Z_{a}} \phi_{s}\left(u^{a} u_{a}\right) d u_{+}^{a} d u_{a}=\int_{Z^{a}} \phi_{s}\left(u^{a} y w_{a}\right) d u_{+}^{a} \times \int_{U_{(a)} \backslash Z_{a}} \phi_{s}\left(\left(\Pi_{\nu}\right)^{r}\right) d u_{a} .
$$

Now the fact that the unipotent group $U_{(a)}(0)$ normalize $U_{+}^{(a)}(0)((26))$, $w_{a} U_{+}^{(a)}(0) w_{a}^{-1}=U_{+}^{(a)}(0)$ and $w_{a} U_{(a)}(0) w_{a}^{-1}=U_{(-a)}(0)$ (by (18)) yields that $U_{(-a)}(0)$ normalize $U_{+}^{(a)}(0)$, and hence the group $x^{\prime} U_{(-a)}(0) x^{\prime^{-1}}$ normalize the group $Z^{a}=x^{\prime} U_{+}^{(a)}(0) x^{\prime-1}$. Here the matrix $y$ in (65) is contained in $K$ and 


$$
y \in U_{(-a)}(r) \subset U_{(-a)}(l)=x^{\prime} U_{(-a)}(0) x^{\prime^{-1}},
$$

we have

$$
\int_{Z^{a}} \phi_{s}\left(u^{a} y w_{a}\right) d u_{+}^{a}=\int_{Z^{a}} \phi_{s}\left(u^{a} w_{a}\right) d u_{+}^{a}
$$

which proves our lemma. For $a=\varepsilon_{\imath}-\varepsilon_{i+1}$, we can prove similarly.

Now, we can give the following relations of $J_{w}^{\prime}(s), J_{w}(s), J_{w^{\prime}}(s)$, and $C_{0}(a, s)$.

Proposition 1. In the notation as before, we have

$$
J_{w}^{1}(s)=C_{0}(a, s) J_{w^{\prime}}\left(w_{a} s\right),
$$

and

$$
J_{w}^{0}(s)=\left(C_{0}(a, s)-1\right) J_{w^{\prime}}(s),
$$

where $w=w^{\prime} w_{a}, l(w)=l\left(w^{\prime}\right)+l\left(w_{a}\right), w_{a}$ is a simple reflection corresponding to the simple root $a \in \Sigma^{+}$.

Proof. Firs let us prove (66). Recall that

$$
J_{w}^{1}(s)=w\left(s \delta^{-1 / 2}\right)(x) \int_{Z^{a}} \int_{U_{(a)}} \phi_{s}\left(u^{a} u_{a}\right) d u_{+}^{a} d u_{a} .
$$

For $u^{a} \in Z^{a}, u_{a} \in U_{(a)}$, we write $w_{a}^{-1} u^{a} w_{a}=k h_{1} n, w_{a} n w_{a}^{-1}=u^{-a} u_{a}^{\prime}$ where $u^{-a}$ $\in U_{-}^{(-a)}$ and $u_{a}^{\prime} \in U_{(a)}$, and put $h_{2}=w_{a} h_{1} w_{a}^{-1} \in H$. Writing $u^{a} u_{a}=w_{a} k h_{1} n w_{a}^{-1} u_{a}$ $=w_{a} k w_{a}^{-1} h_{2} w_{a} n w_{a}^{-1} u_{a}$, we have

$$
\phi_{s}\left(u^{a} u_{a}\right)=\phi_{s}\left(h_{2} w_{a} n w_{a}^{-1} u_{a}\right)=\phi_{s}\left(h_{2} u^{-a} u_{a}^{\prime} u_{a}\right) .
$$

Since $u^{-a} u_{a}^{\prime} u_{a}=\left(u_{a}^{\prime} u_{a}\right)\left\{w_{a}\left(w_{a} u_{a}^{\prime} u_{a} w_{a}\right)^{-1}\left(w_{a} u^{-a} w_{a}\right)\left(w_{a} u_{a}^{\prime} u_{a} w_{a}\right) w_{a}\right\} \in\left(u_{a}^{\prime} u_{a}\right) U_{-}^{(-a)}$ we have (69) $=\phi_{s}\left(h_{2} u_{a}^{\prime} u_{a}\right)$ and (using (11)) it is equal to

$$
\phi_{s}\left(h_{2} u_{a}^{\prime} u_{a} h_{2}^{-1} h_{2}\right)=\phi_{s}\left(h_{2} u_{a}^{\prime} u_{a} h_{2}^{-1}\right)\left(s \delta^{1 / 2}\right)\left(h_{2}\right) .
$$

Here we have (using (34) and $w_{a}^{2}=1$ )

$$
\left(s \delta^{1 / 2}\right)\left(h_{2}\right)=s\left(w_{a} h_{1} w_{a}^{-1}\right) \delta^{1 / 2}\left(h_{2}\right)=\phi_{w_{a} s}\left(w_{a}^{-1} u^{a} w_{a}\right) \delta_{a}\left(h_{2}\right)
$$

Thus we have

$$
\phi_{s}\left(u^{a} u_{a}\right)=\phi_{s}\left(h_{2} u_{a}^{\prime} u_{a} h_{2}^{-1}\right) \times \phi_{w_{a} s}\left(w_{a}^{-1} u^{a} w_{a}\right) \delta_{a}\left(h_{2}\right) .
$$


Hence we have

$$
\begin{aligned}
& \int_{Z^{a}} \int_{U_{(a)}} \phi_{s}\left(u^{a} u_{a}\right) d u_{+}^{a} d u_{a}=\int_{Z^{a}} \int_{U_{(a)}} \phi_{s}\left(h_{2} u_{a}^{\prime} u_{a} h_{2}^{-1}\right) \phi_{w_{a} s}\left(w_{a}^{-1} u^{a} w_{a}\right) \delta_{a}\left(h_{2}\right) d u_{+}^{a} d u_{a} \\
& =\int_{U_{(a)}} \phi_{s}\left(u_{a}\right) d u_{a} \int_{w_{a}^{-1} Z^{a} w_{a}} \phi_{w_{a} s}\left(u^{a}\right) d u^{a}=C_{0}(a, s) \int_{w_{a}^{-1} Z^{a} w_{a}} \phi_{w_{a} s}\left(u^{a}\right) d u^{a}
\end{aligned}
$$

Substituting this to (68),

$$
J_{w}^{1}(s)=w\left(s \delta^{-1 / 2}\right)(x) C_{0}(a, s) \int_{w_{a}^{-1} Z^{a} w_{a}} \phi_{w_{a} s}\left(u^{a}\right) d u_{+}^{a} .
$$

By Lemma 1, we have

$$
\begin{gathered}
J_{w^{\prime}}\left(w_{a} s\right)=w\left(s \delta^{-1 / 2}\right)(x) \int_{w_{a}^{-1} Z^{a} w_{a}} \phi_{w_{a} s}\left(u^{a}\right) d u_{+}^{a}, \text { and hence } \\
J_{w}^{1}(s)=C_{0}(a, s) J_{w^{\prime}}\left(w_{a} s\right)
\end{gathered}
$$

Now we have completed the proof of (66). Next we prove (67).

First, it is easy to see from Lemma 1 that

$$
\begin{gathered}
\int_{Z^{a}} \phi_{s}\left(u^{a} w_{a}\right) d u_{+}^{a}=\int_{Z^{a}} \phi_{s}\left(w_{a}^{-1} u^{a} w_{a}\right) d u_{+}^{a} \\
=\int_{w_{a}^{-1} Z^{a} w_{a}} \phi_{s}\left(u^{a}\right) d u_{+}^{a}=\left(w^{\prime} s(x)\right)^{-1}\left(w \delta^{1 / 2}\right)(x) J_{w^{\prime}}(s) .
\end{gathered}
$$

By definition

$$
\begin{aligned}
& J_{w}^{0}(s)=w\left(s \delta^{-1 / 2}\right)(x) \int_{Z^{a}} \int_{U_{(a)} \backslash Z_{a}} \phi_{s}\left(u^{a} u_{a}\right) d u_{+}^{a} d u_{a} \\
= & w\left(s \delta^{-1 / 2}\right)(x) \int_{Z^{a}} \phi_{s}\left(u^{a} w_{a}\right) d u_{+}^{a} \cdot \int_{U_{(a)} \backslash Z_{a}} \phi_{s}\left(u_{a}\right) d u_{a} \text { (by Lemma 4) } \\
= & w\left(s \delta^{-1 / 2}\right)(x)\left(w^{\prime} s(x)\right)^{-1}\left(w \delta^{1 / 2}\right)(x) J_{w^{\prime}}(s) \int_{U_{(a)} \backslash z_{a}} \phi_{s}\left(u_{a}\right) d u_{a} \\
= & w s(x) \cdot\left(w^{\prime} s(x)\right)^{-1} \cdot J_{w^{\prime}}(s) \int_{U_{(a)} \backslash Z_{a}} \phi_{s}\left(u_{a}\right) d u_{a} .
\end{aligned}
$$

Now using Lemma 3 , we have $J_{w}^{0}=\left(C_{0}(a, s)-1\right) J_{w^{\prime}}(s)$.

2.3. A character $s$ of $D$ is called non-singular if $s\left(\Pi_{i}\right) \neq 1$ for all $i \neq 1, \ldots$, $\nu-1$, and $\left|s\left(\Pi_{\nu}\right)\right| \neq 1$. Now we prove the following proposition. 
Proposition 2 (reduction formula). Suppose that $s$ is nonsingular and $a \in \Sigma^{+} a$ simple root such that wa is a negative root and $w^{\prime}=w w_{a}$.

$$
J_{w}(s)=C_{0}(a, s) J_{w^{\prime}}\left(w_{a} s\right)-\left(C_{0}(a, s)-1\right) J_{w^{\prime}}(s)
$$

Proof. The formula in our proposition follows from Proposition 1, as far as the integrals are convergent, and it is satisfied if $|s(\Pi(a))|>1$. Now $J_{w}(s)$, $J_{w^{\prime}}(s)$, and $J_{w^{\prime}}\left(w_{a} s\right)$ are polynomials with respect to $s\left(d_{i}\right) d_{i} \in D$ as mentioned before. Therefore (73) is valid (by analytic continuation) whenever the right-hand side is defined, i.e., whenever $s$ is nonsingular.

For $w=w_{1} \ldots w_{r} \in W$ (reduced word), we denote by $E(w)$ the set of all products $w_{1}^{\varepsilon_{1}} \cdots w_{r}^{\varepsilon_{r}}\left(\varepsilon_{i}=0\right.$ or 1$)$.

Lemma 5. Suppose that a character $s$ of $D$ is non-singular. Then

$$
J_{w}(s)=\delta^{1 / 2}(x) \sum_{\tilde{w} \in E(w)} \lambda_{w, \tilde{w}}(s)(\tilde{w} s)(x) .
$$

Here the coefficients $\lambda_{w, \tilde{w}}(s)$ are rational functions which do not dependent on $x$. Moreover we have

$$
\lambda_{w, w}(s)=\prod_{a \in \Sigma_{w}^{+}} C_{0}(a, s)
$$

where $\Sigma_{w}^{+}$is the set of all roots $a \in \Sigma^{+}$such that wa is negative.

Proof. We prove this lemma by induction on the length $l(w)=r$ of $w$. First of all, if $r=1$, that is to say $w=w_{a}$, then we have, $E(w)=\{1, w\}$ and $J_{w}(s)=$ $C_{0}(a, s) J_{1}(w s)-\left(C_{0}(a, s)-1\right) J_{1}(s)$ by Proposition $2(1$ means identity of the group $W$. Since $J_{1}(s)=s \delta^{1 / 2}(x)$ from (45), we have

$$
\begin{aligned}
& J_{w}(s)=C_{0}(a, s) \cdot w s(x) \cdot \delta^{1 / 2}(x)-\left(C_{0}(a, s)-1\right)\left(s \delta^{1 / 2}\right)(x) \\
= & \delta^{1 / 2}(x)\left(C_{0}(a, s) \cdot(w s)(x)-\left(C_{0}(a, s)-1\right) \cdot s(x)\right) .
\end{aligned}
$$

Therefore $\lambda_{w, w}=C_{0}(a, s)$, and $\lambda_{w, 1}=C_{0}(a, s)-1$. Thus we have proved the case where $r=1$.

Next, suppose $r=m$ and assume our lemma is true if $l\left(w^{\prime}\right) \leq m-1 \quad w^{\prime} \in$ $W$. We write $w$ as $w=w^{\prime} w_{a}, l\left(w^{\prime}\right)=m-1$, and $l\left(w_{a}\right)=1$. Applying the induction assumption to $w^{\prime}$, we have

$$
J_{w^{\prime}}(s)=\delta^{1 / 2}(x) \sum_{\tilde{w} \in E\left(w^{\prime}\right)} \lambda_{w^{\prime}, \tilde{w}}(s) \cdot(\tilde{w} s)(x), \quad \lambda_{w^{\prime}, w^{\prime}}(s)=\prod_{b \in \Sigma_{w}^{+}} C_{0}(b, s) .
$$


Putting this into the formula in Proposition 2,

$$
\begin{aligned}
J_{w}(s) & =C_{0}(a, s) \delta^{1 / 2}(x) \sum_{\tilde{w} \in E\left(w^{\prime}\right)} \lambda_{w^{\prime}, \tilde{w}}\left(w_{a} s\right) \cdot\left(\tilde{w} w_{a} s\right)(x) \\
& -\left(C_{0}(a, s)-1\right) \delta^{1 / 2}(x) \sum_{\tilde{w} \in E\left(w^{\prime}\right)} \lambda_{w^{\prime}, \tilde{w}}(s) \cdot(\tilde{w} s)(x) .
\end{aligned}
$$

Since $E\left(w^{\prime}\right) \subset E(w), E\left(w^{\prime}\right) w_{a} \subset E(w)$, the equality above implies that $J_{w}(s)$ has a required form. Moreover since $\lambda_{w, w}$ is the coefficient of $(w s)(x)=$ $\left(\left(w^{\prime} w_{a}\right)(s)(x)\right)$ we have

$$
\lambda_{w, w}=C_{0}(a, s) \cdot \lambda_{w^{\prime}, w^{\prime}}\left(w_{a} s\right)=C_{0}(a, s) \prod_{b \in \Sigma_{w^{\prime}}^{+}} C_{0}\left(b, w_{a} s\right) .
$$

Since $w_{a}\left(\sum_{w^{\prime}}^{+}\right) \cup\{a\}=\sum_{w}^{+}$, we have from $(63) \lambda_{w, w}(s)=\prod_{a \in \Sigma_{w}^{+}} C_{0}(a, s)$.

Substituting the formula of Lemma 5 to (44), have .

$$
\omega_{s}\left(x^{-1}\right)=\delta^{1 / 2}(x) \cdot \sum_{w \in W} V_{w} \cdot \mu_{w}(s) \cdot(w s)(x),
$$

where $\mu_{w}(s)$ are independent of $x$. It follows from Lemma 5 that

$$
\mu_{w_{0}}(s)=\lambda_{w_{0}, w_{0}}(s)=\prod_{a \in \Sigma^{+}} C_{0}(a, s),
$$

where $w_{0}$ is the longest element of $W$. By virtue of $\omega_{s}\left(x^{-1}\right)=\omega_{\tilde{w} s}\left(x^{-1}\right)$ for all $\tilde{w} \in W([7$, p.26]), (76) implies

$$
\sum_{w} V_{w} \mu_{w}(\tilde{w} s)(w \tilde{w} s)(x)=\sum_{w} V_{w} \mu_{w}(s)(w s)(x) \text { for all } \tilde{w} \in W .
$$

Now from the linearly independence of characters $w s$ over $\mathbf{C}$ for $w \in W$, we have $V_{w} \mu_{w}(s)=V_{1} \mu_{1}(w s)$, especially we have (using the fact that $C_{0}\left(a, w_{0} s\right)=C_{0}(a$, $\left.s^{-1}\right)$, which is obtained from the definition of $C_{0}(a, s)$ and $w_{0}^{-1} \Pi_{i} w_{0}=$ $\left.\Pi_{\imath}^{-1}\right) V_{w_{0}} \mu_{w_{0}}(s)=V_{1} \mu_{1}\left(w_{0} s\right)=V_{1} \mu_{1}\left(s^{-1}\right)$. This implies $V_{w_{0}} \mu_{w_{0}}\left(s^{-1}\right)=V_{1} \mu_{1}(s)$. Then we have

$$
V_{w} \mu_{w}(s)=V_{1} \mu_{1}(w s)=V_{w_{0}} \mu_{w_{0}}\left(w s^{-1}\right)=V_{w_{0}} \cdot \prod_{a \in \Sigma^{+}} C_{0}\left(a, w s^{-1}\right)=V_{w_{0}} C\left(w s^{-1}\right),
$$

where we put $C(s)=\prod_{a \in \Sigma^{+}} C_{0}(a, s)$. Thus we have

$$
\sum V_{w} \mu_{w}(s)(w s)(x)=V_{w_{0}} \sum C\left(w s^{-1}\right)(w s)(x),
$$

and then we have proved the following theorem. (Except for the case where $\alpha=0$, the theorem below is obtained by Casselman [4].) 
THEOREm 2 (Formulae for spherical functions of orthogonal groups). Suppose $s$ is nonsingular. Then, for

$$
x=\Pi^{(m)},(m)=\left(m_{1}, \ldots, m_{\nu}, \frac{m_{0}}{e_{0}}\right) \text { with } m_{1} \geq \cdots \geq m_{\nu} \geq \frac{1}{e_{0}} m_{0},
$$

the spherical function is given as follows:

$$
\omega_{s}\left(x^{-1}\right)=\kappa \delta^{1 / 2}(x) \sum_{w \in W} C\left(w s^{-1}\right)(w s)(x),
$$

where

$$
C(s)=\prod_{a \in \Sigma^{+}} C_{0}(a, s),
$$

$C_{0}(a, s)$ are given in Theorem 1 , and $\kappa$ is a constant.

We calculate the value of $\kappa$. Substituting $s=\delta^{-1 / 2}$ to the formula of the theorem above, we have

$$
1=\kappa \delta^{1 / 2}(x) \sum_{w \in W} C\left(w s^{-1}\right)(w s)(x)=\kappa C\left(\delta^{1 / 2}\right),
$$

where we used Remark 6. This yields $\kappa=C\left(\delta^{1 / 2}\right)^{-1}$.

\section{§3. Calculations of the volume of $K x K$}

In the notation as before, let $x=\Pi^{(m)} \in D$ such that

$$
(m)=\left(m_{1}, \ldots, m_{\nu}, \frac{m_{0}}{e_{0}}\right) \text { with } m_{1} \geq \cdots \geq m_{\nu} \geq \frac{1}{e_{0}} m_{0} .
$$

By (19) and (39), we have $B w B=B w B^{-1}=B w \cdot U^{-}(1) H_{0} U^{+}(0)=B w U^{+}(0)$, where we used $w U^{-}(1) H_{0} w^{-1}=w U^{-}(1) w^{-1} w H_{0} w^{-1} \subset B$ (by (38)). Then by virtue of Theorem $\mathrm{H}$, we have

$$
\begin{gathered}
K x K=K w_{0} x K=\bigcup_{w \in W} B w U^{+}(0) w_{0} x K=\bigcup_{w \in W} B w \cdot w_{0} x K \\
=\bigcup_{w \in W} B w x K=\bigcup_{w \in W} B \cdot w x w^{-1} K
\end{gathered}
$$

(This is not necessarily a disjoint union.) where we used $\left(w_{0} x\right)^{-1} U^{+}(0)\left(w_{0} x\right)=$ $x^{-1} U^{-}(0) x \subset U^{-}(0) \subset K$, (by (37)) and $w_{0}$ is the longest element of $W$. We define $W_{x}$ by $W_{x}:=\left\{w \in W \mid w x w^{-1}=x\right\}\left(=\{w \in W \mid w m=m\}, x=\Pi^{(m)}\right)$. Then, it is trivial that $B w w^{\prime} x\left(w w^{\prime}\right)^{-1} K=B w x w^{-1} K$ for all $w^{\prime} \in W_{x}$. Therefore we have 


$$
K x K=\bigcup_{w \in W / w_{x}} B w x w^{-1} K
$$

Let us see that (79) is a disjoint union. If $B w x w^{-1} K \cap B w^{\prime} x w^{-1} K \neq\{\phi\}$ for some $w, w^{\prime} \in W$, there is an element $b_{0} \in B$ such that $\left(w^{\prime} x w^{\prime-1}\right)^{-1} b_{0}\left(w x w^{-1}\right) \in$ $K$. Thus diagonal entries of $\left(w^{\prime} x w^{\prime^{-1}}\right)^{-1}\left(w x w^{-1}\right)$ must be unit elements. This implies $w^{\prime} x w^{\prime-1}=w x w^{-1}$, and hence (79) is a disjoint union. Thus we have

$$
|K x K / K|=\frac{1}{\left|W_{x}\right|} \sum_{w \in W}|B w x K / K|
$$

where $|*|$ denotes the number of the elements of a finite set $*$. There exists a unique set $W_{\text {rep }}$ of representatives of $W / W_{x}$ with the property that each $w \in W_{\text {rep }}$ is of minimal length in the coset $w W_{x}$. Then, each $w \in W_{\text {rep }}$ satisfies a relation

$$
l\left(w w^{\prime}\right)=l(w)+l\left(w^{\prime}\right),
$$

for any $w^{\prime} \in W_{x}$. Now denote by $l^{\prime}(w)$ the number of $w_{e_{\nu}}$ in the shortest expression of $w$ with respect to simple reflections. Then

$$
l^{\prime}\left(w w^{\prime}\right)=l^{\prime}(w)+l^{\prime}\left(w^{\prime}\right) \text { for all } \quad w \in W_{\text {rep }} \text { and } \quad w^{\prime} \in W_{x} .
$$

We put

$$
L(w)=l(w)+(\alpha-1) l^{\prime}(w) .
$$

Then (81), and (82) implies

$$
L\left(w w^{\prime}\right)=L(w)+L\left(w^{\prime}\right) \text { for all } \quad w \in W_{\text {rep }} \text { and } \quad w^{\prime} \in W_{x} .
$$

Using (21), and (62), we see that

$$
q^{L\left(w_{a}\right)}=\left[U_{(a)}(0): U_{(a)}(1)\right]=\left[U_{(-a)}(0): U_{(-a)}(1)\right]
$$

for any simple reflection $w_{a} \in W$.

Now the following lemma holds.

Lemma 6 . In the natation as before,

$$
|B w x K / K|=q^{-L(w)}|B x K / K| \text { for all } w \in W_{\text {rep }} .
$$

Proof. We prove this lemma by induction on the length of $w \in W_{\text {rep }}$. If $l(w)=0$, then $w=1$ and hence the equation above is clear. Now we assume that our lemma is valid for $\tilde{w} \in W_{\text {rep }}$ such that $l(\tilde{w}) \leq l(w)-1$. Let $w_{a}$ be a simple 
reflection such that $l\left(w_{a} w\right)=l(w)-1$. We put $w^{\prime}=w_{a} w$. Now if $l\left(w^{\prime}\right)>$ $l\left(w^{\prime} w_{1}\right)$ for some $w_{1} \in W$, then $l(w)=l\left(w^{\prime}\right)+1>l\left(w^{\prime} w_{1}\right)+1 \geq l\left(w_{a} w^{\prime} w_{1}\right)=$ $l\left(w w_{1}\right)$, which implies that $w \in W_{\text {rep }}$ means $w^{\prime} \in W_{\text {rep }}$. Thus we have by assumption,

$$
\left|B w^{\prime} x K / K\right|=q^{-L\left(w^{\prime}\right)}|B x K / K| .
$$

Now (19), (26), (27) implies for a simple root $a$,

$$
B=U^{+}(0) H_{0} U^{-}(1)=U_{+}^{(a)}(0) \cdot U_{(a)}(0) \cdot H_{0} \cdot U_{(-a)}(1) \cdot U_{-}^{(-a)}(1) .
$$

It is easy to see

$$
U_{(-a)}(1) \cdot U_{+}^{(a)}(0)=U_{+}^{(a)}(0) \cdot U_{(-a)}(1)
$$

and

$$
U_{(a)}(0) H_{0} U_{(-a)}(1)=U_{(-a)}(1) H_{0} U_{(a)}(0) .
$$

Therefore from (85), (86), (87), we have

$$
\begin{aligned}
B & =U_{+}^{(a)}(0) U_{(-a)}(1) H_{0} U_{(a)}(0) U_{-}^{(a)}(1) \\
& =U_{(a)}(1) \cdot U_{+}^{(a)}(0) \cdot H_{0} \cdot U_{-}^{(-a)}(1) \cdot U_{(a)}(0) .
\end{aligned}
$$

From this, we have

$$
\begin{aligned}
B w x K & =U_{(-a)}(1) \cdot U_{+}^{(a)}(0) \cdot H_{0} \cdot U_{-}^{(-a)}(1) \cdot U_{(a)}(0) \cdot w x K \\
& =U_{(-a)}(1) \cdot U_{+}^{(a)}(0) \cdot U_{-}^{(-a)}(1) \cdot w x K .
\end{aligned}
$$

On the other hand, we have from (85)

$$
\begin{gathered}
w_{a} \cdot B w^{\prime} x K=w_{a} \cdot U_{(a)}(0) \cdot U_{+}^{(a)}(0) \cdot H_{0} \cdot U_{-}^{(-a)}(1) \cdot U_{(-a)}(1) w^{\prime} x K \\
=w_{a} \cdot U_{(a)}(0) \cdot U_{+}^{(a)}(0) \cdot H_{0} \cdot U_{-}^{(-a)}(1) \cdot w^{\prime} x K,\left(\text { by } w^{\prime^{-1}}(-a) \in \Sigma^{-},(12) \text { and }(37)\right) \\
=U_{(-a)}(0) \cdot U_{+}^{(a)}(0) \cdot U_{-}^{(-a)}(1) \cdot w x \cdot K .
\end{gathered}
$$

From (89) and (90), we have

$$
w_{a} \cdot B w^{\prime} x K,=\bigcup_{u} u \cdot B w x K
$$

where $u$ runs through a complete set of representatives of $U_{(-a)}(0) / U_{(-a)}(1)$. Now let us show that $u \cdot B w x K \cap B w x K \neq\{\phi\}$ for some $u \in U_{(-a)}(0)$ implies $u \in$ $U_{(-a)}(1)$. If $u \cdot B w x K \cap B w x K \neq\{\phi\}$ for some $u \in U_{(-a)}(0)$, then there exists $b \in B$ such that

$$
u \cdot b w x K \cap U_{(-a)}(1) U_{+}^{(a)}(0) U_{-}^{(-a)}(1) \cdot w x K \neq\{\phi\} .
$$


Now since $U_{(-a)}(0)$ normalize $U_{+}^{(a)}(0)$ and $U_{-}^{(-a)}(1)$, and the groups $U_{(-a)}(1)$, $U_{+}^{(a)}(0)$, and $U_{-}^{(-a)}(1)$ are all contained in the group $B$, we have from (91), $u \cdot \tilde{b} \in$ $(w x) K(w x)^{-1}$ for some $\tilde{b} \in B$, i.e.,

$$
\left(w x w^{-1}\right)^{-1}(u \cdot \tilde{b})\left(w x w^{-1}\right) \in K \text { for some } \tilde{b} \in B .
$$

Now since $w \in W_{\text {rep }}$, and $w^{\prime}=w_{a} w \in W_{\text {rep }}$, we have, putting $w x w^{-1}=\Pi^{(m)} m \neq$ $w_{a}(m)$. Therefore we have

$$
\operatorname{ord}_{\mathfrak{p}}\left(w x w^{-1}\right)^{-1} u\left(w x w^{-1}\right) \neq \operatorname{ord}_{\mathfrak{p}} u \quad \text { for } \quad u \in U_{(-a)} .
$$

Moreover since $w^{-1}(-a) \in \Sigma^{+}$, we have

$$
\operatorname{ord}_{\mathfrak{p}}\left(w x w^{-1}\right)^{-1} u\left(w x w^{-1}\right) \leq \operatorname{ord}_{\mathfrak{p}} u \quad \text { for } \quad u \in U_{(-a)} .
$$

From (93) and (94) we have

$$
\operatorname{ord}_{\mathfrak{p}}\left(w x w^{-1}\right)^{-1} u\left(w x w^{-1}\right)<\operatorname{ord}_{\mathfrak{p}} u \quad \text { for } \quad u \in U_{(-a)} .
$$

Therefore (92) can hold only if $u \in B$, i.e., $u \in U_{(-a)}$ (1). Thus we have

$$
\begin{gathered}
\left|B w^{\prime} x K / K\right|=\left|w_{a} B w^{\prime} x K / K\right|=|B w x K / K| \times\left[U_{(-a)}(0): U_{(-a)}(1)\right] \\
=q^{L\left(w_{a}\right)}|B w x K / K| .
\end{gathered}
$$

Therefore, using the induction assumption (84), we have

$$
|B w x K / K|=q^{-L\left(w_{a}\right)} \cdot\left|B w^{\prime} x K / K\right|=q^{-L\left(w_{a}\right)-L\left(w^{\prime}\right)}|B x K / K|=q^{-L(w)}|B x K / K| .
$$

Now we have proved our lemma.

Now we have from Lemma 6 ,

$$
q^{-L\left(w^{\prime}\right)} \times \sum_{w \in W}|B w x K / K|=|B x K / K| \times\left|W_{x}\right| \times \sum_{w \in W_{\text {rep }}} q^{-L\left(w w^{\prime}\right)},
$$

for all $w^{\prime} \in W_{x}$. Thus we have

$$
\sum_{w^{\prime} \in W_{x}}\left(q^{-L\left(w^{\prime}\right)}\right) \times \sum_{w \in W}|B w x K / K|=|B x K / K| \times\left|W_{x}\right| \times \sum_{w \in W} q^{-L(w)}
$$

This implies

$$
\begin{aligned}
& |K x K / K| \frac{1}{\left|W_{x}\right|} \sum_{w \in W}|B w x K / K| \quad(\text { by }(80)) \\
= & |B x K / K| \cdot \sum_{w \in W} q^{-L(w)} \cdot\left(\sum_{w^{\prime} \in W_{x}} q^{-L\left(w^{\prime}\right)}\right)^{-1}
\end{aligned}
$$

Now we calculate $|B x K / K|$. Since 


$$
\begin{gathered}
B x K=U^{+}(0) H_{0} U^{-}(1) x K \quad(\text { by (19)) } \\
=U^{+}(0) x x^{-1} H_{0} U^{-}(1) x K=U^{+}(0) x K, \quad\left(\text { by }(37) \text { and } x^{-1} H_{0} x=H_{0}\right)
\end{gathered}
$$

we have

$$
\begin{gathered}
|B x K / K|=\left|x^{-1} B x K / K\right|=\left|x^{-1} U^{+}(0) x K / K\right| \\
=\left|x^{-1} U^{+}(0) x / K \cap x^{-1} U^{+}(0) x\right|=\left|x^{-1} U^{+}(0) x / U^{+}(0)\right| \quad \text { (by (37)) } \\
=\delta(x) \text { (see (8)). }
\end{gathered}
$$

Putting (95) into (96), we have the following theorem.

THEOREM 3. In the notation as before, we have

$$
|K x K / K|=\delta(x) \cdot \sum_{w \in W} q^{-L(w)} \cdot\left(\sum_{w^{\prime} \in W_{x}} q^{-L\left(w^{\prime}\right)}\right)^{-1} .
$$

\section{REFERENCES}

[1] A. N. Andriaov, Spherical functions for $G L_{n}$ over local fields, and summation of Hecke series, Math USSR Sbornik., 12 (1970), 429-451.

[2] A. N. Andrianov, On factorization of Hecke polynomials for the symplectic group of genus $n$, Math USSR Sbornik., 33 (1977), 343-373.

[3] F. Bruhat and J. Tits, Groupes reductifs sur un corps local, Publ. Math. Inst. Hautes Etudes Sci., 41 (1972), 5-251.

[4] W. Casselman, The unramified principal series of $p$-adic groups I, The spherical function, Compositio Math., 40 (1980), 387-406.

[ 5 ] H. Hijikata, Maximal compact subgroups of some $\mathfrak{p}$-adic classical groups, Yale University.

[6] I. G. Macdonald, Spherical functions on a group of $p$-adic type, Advanced Study of Math. Madras., (1970).

[7] I. Satake, Theory of spherical functions on reductive algebraic groups over $p$-adic fields, I.H.E.S., 18 (1963).

[8] T. A. Springer, Linear algebraic groups, Birkhauser.

[9] J. Tits, Reductive groups over local fields, Proc. of Sympoia in Pure Math., 33 part 1 , A.M.S., (1979), 29-69.

Graduate School of Polymathematics

Nagoya University

Chikusa-ku, Nagoya, 464-01,

Japan 